\title{
Modelamiento numérico del embalse Murallas Viejas. Efectos de incorporar la pantalla de hormigón
}

\author{
Numerical analysis of Murallas Viejas dam. Effects assessment when accounting for the \\ concrete face
}

Fecha de entrega: 18 de diciembre 2018 Fecha de aceptación: 2 de mayo 2019

\section{Claudia Vallejos ${ }^{1}$ y Alfredo Arenas ${ }^{2}$ \\ ${ }^{1}$ Arcadis, Antonio Varas 621, piso 6, Providencia, Región Metropolitana de Santiago, Chile, claudia.vallejos@arcadis.com \\ 2 Golder Associates, Magdalena 181, piso 3, Las Condes, Región Metropolitana de Santiago, Chile, clvallejos@golder.cl, aarenas@golder.cl}

Como parte de los diseños del embalse Murallas Viejas, se evaluó la respuesta dinámica de la presa de enrocados de $130 \mathrm{~m}$ de altura. Durante esta evaluación se modeló la interacción entre la pantalla de hormigón y el muro de enrocados. Para la representación de la pantalla de hormigón se utilizaron técnicas avanzadas de modelación, permitiendo capturar los efectos inerciales, su resistencia y su capacidad de deformarse, asi como también la interacción que existe entre las losas que la componen y la condición de borde que ejercería el plinto durante cargas sísmicas. Los resultados de la modelación numérica realizada para el proyecto Murallas Viejas, fueron comparados con un análisis análogo, pero sin incluir la pantalla de hormigón directamente en los modelos numéricos. Para este caso se obtuvo la respuesta de la pantalla de hormigón en forma indirecta, mediante post análisis. El presente trabajo muestra los efectos de la pantalla sobre las deformaciones de la presa, las técnicas de modelación utilizadas y destaca cuales fueron los puntos claves para su implementación. Los resultados indican que la respuesta de la presa, y en especial los momentos generados en la pantalla de hormigón, difieren sustancialmente entre ambos casos. Los autores creen que la diferencia se ve magnificada por el hecho de incorporar en los análisis los materiales de transición.

Palabras clave: CFRD, embalse, respuesta sísmica, FLAC $3 D$
During the dynamic analysis of the Murallas Viejas reservoir, the response of the rockfill dam of $130 \mathrm{~m}$ high was evaluated, during this evaluation, the interaction between the concrete face and the rockfill dam was modeled using advanced techniques. This implementation allowed capturing the concrete face response in term of inertial effects, imposed stresses and its ability for deforming, as well as capturing the slab interaction with each other, the interaction with the rockfill dam and capturing the boundary effects imposed by the plinth. The results of the numerical modelling carried out for the Murallas Viejas reservoir were compared with a similar analysis, but without including the concrete face in the numerical models. For this case the response of the concrete face was indirectly obtained, by a post analysis. The results illustrate the complex interaction between the different dam components and the need of using a correct technique for modelling the dam system. The present work shows the various techniques used and highlight key points of its implementation. The results indicate that the dam response, especially those related to flexural moments at the concrete face, significantly differ between the two cases. The authors believe that this difference is magnified by the inclusion of the transition materials in the numerical analysis.

Keywords: CFRD, reservoir, seismic response, FLAC3D

\section{Introducción}

La Dirección de Obras Hidráulicas (DOH) desarrolló el proyecto Embalse Murallas Viejas con el objetivo de aumentar la disponibilidad de recursos hídricos para riego en la región de Coquimbo. El embalse se ubicará en el río Combarbalá y consistirá de una presa del tipo CFRD (por su sigla en inglés, Concrete Face Rockfill Dam).

La presa se conformará de material de enrocado compactado 
y se impermeabilizará con una pantalla de hormigón en la cara aguas arriba, la cual estará conectada a un plinto en toda su extensión en contacto con la roca de fundación. El embalse tendrá una capacidad de acumular $50 \mathrm{Mm}^{3}$.

Para evaluar la estabilidad dinámica del muro de enrocados durante la ocurrencia de solicitaciones sísmicas, se realizó un modelo numérico tridimensional, mediante el uso del software FLAC3D versión 5.01 (Cundall, 2016), en el que se estudiaron 2 escenarios: condición embalse vacío y condición embalse lleno. Este estudio hace referencia a la condición de embalse vacío.

En la práctica, los análisis numéricos para este tipo de estructuras se realizan considerando la presa sin la acción de la pantalla, por lo que la interacción muro-pantalla se logra imponiendo los desplazamientos del muro sobre la pantalla mediante post análisis estructural, y con ello obtener los momentos actuantes sobre ella. Esta metodología puede subestimar los esfuerzos actuando sobre la pantalla, por lo que el presente análisis considera la interacción dinámica muro-pantalla.

De esta manera, el análisis numérico incluyó la modelación de la geometría del embalse, caracterización geotécnica de los materiales, la utilización de modelos constitutivos no lineales, la simulación del proceso constructivo del muro y su interacción con los elementos estructurales que forman la pantalla de hormigón y el plinto.

\section{Descripción de la presa}

La presa tendrá una altura máxima de $130 \mathrm{~m}$, con ancho y largo de coronamiento de $8 \mathrm{~m}$ y $576 \mathrm{~m}$, respectivamente. La geometría está conformada con taludes aguas arriba 1.5:1 $(\mathrm{H}: \mathrm{V})$ y variable aguas abajo de 1.6:1 $(\mathrm{H}: \mathrm{V})$ en el tercio superior del muro y 1.4:1 (H:V) en los dos tercios inferiores.

La geometría y mallado 3D del modelo se realizó en el software GeoMeshing versión 2.6.5, el que permite generar el mallado 3D por medio de una combinación de elementos y optimizado para la corrida dinámica. Un total de 680000 zonas fueron generadas en el modelo.

La Figura 1 muestra la geometría 2D de la sección representativa del modelo y su geometría 3D. Para efectos de este trabajo el eje $\mathrm{X}$ se define como la dirección transversal al coronamiento del muro, el eje $\mathrm{Y}$ en la dirección longitudinal y el eje $\mathrm{Z}$ como la dirección vertical.

a)

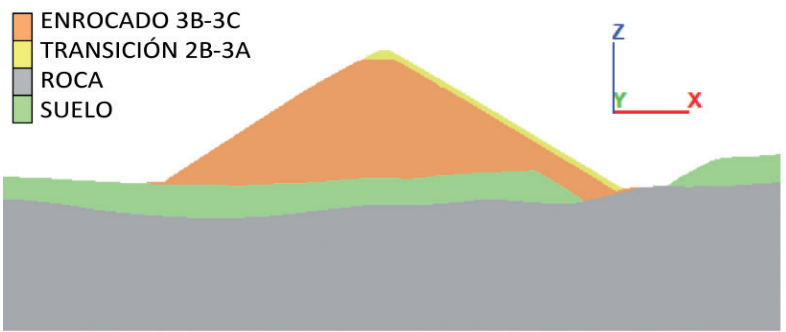

(A) (A

b)

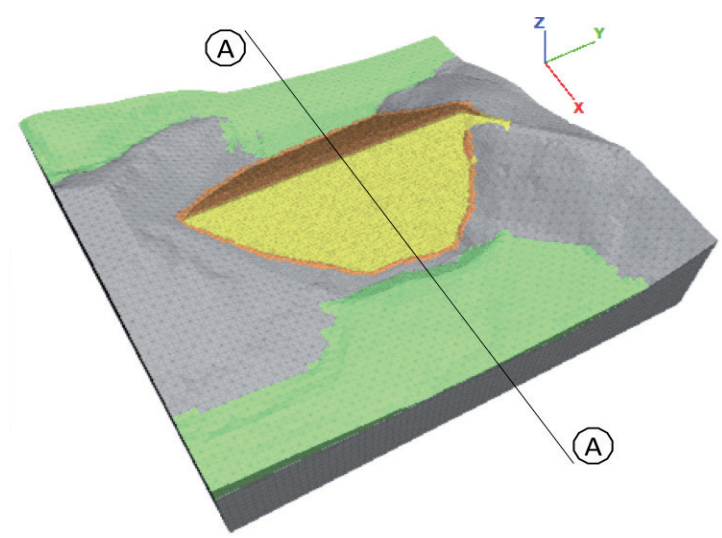

Figura 1: a) Sección transversal de la presa y b) modelo 3D en FLAC

\section{Caracterización geotécnica de los materiales}

Las propiedades geotécnicas de los materiales de la presa modelados para el análisis dinámico se muestran en la Tabla 1 .

Tabla 1: Caracterización geotécnica de los materiales y parámetros dinámicos

\begin{tabular}{|l|c|c|c|c|c|c|c|c|c|}
\hline Material & $\rho, \mathrm{t} / \mathrm{m}^{3}$ & $\eta$ & $S$ & $G_{\mathrm{max}}, \mathrm{kPa}$ & $B_{\max }, \mathrm{kPa}$ & $K_{\mathrm{g}}, K_{\mathrm{b}}$ & $n_{\mathrm{g}}, n_{\mathrm{b}}$ & $\phi,{ }^{\circ}$ & $c, \mathrm{kPa}$ \\
\hline Roca & 2.20 & 0.02 & 1.0 & $6.6 \cdot 10^{6}$ & $1.1 \cdot 10^{7}$ & - & - & - & - \\
\hline Aluvial & 1.88 & - & 0.3 & $K_{\mathrm{g}} P_{\mathrm{a}}\left(p^{\prime} / P_{\mathrm{a}}\right)^{\mathrm{n}_{\mathrm{g}}}$ & $K_{\mathrm{b}} P_{\mathrm{a}}\left(p^{\prime} / P_{\mathrm{a}}\right)^{\mathrm{n}_{\mathrm{b}}}$ & 1316 & 0.75 & 37 & 0 \\
\hline Transición 2B-3A & 2.00 & 0.25 & - & $K_{\mathrm{g}} P_{\mathrm{a}}\left(p^{\prime} / P_{\mathrm{a}}\right)^{\mathrm{n}_{\mathrm{g}}}$ & $K_{\mathrm{b}} P_{\mathrm{a}}\left(p^{\prime} / P_{\mathrm{a}}\right)^{\mathrm{n}} \mathrm{b}$ & 2666 & 0.10 & 40 & 0 \\
\hline Enrocado 3B-3C & 2.10 & 0.25 & - & $f\left(\rho, V_{\mathrm{s}}\right)$ & $G_{\max }$ & - & - & $f\left(p^{\prime} / P_{\mathrm{a}}\right)$ & 0 \\
\hline
\end{tabular}

$\rho$ : densidad seca, $\eta$ : porosidad, $S$ : saturación, $G_{\max }$ : módulo de corte máximo, $B_{\max }$ : módulo volumétrico máximo, $K_{\mathrm{g}}$ : factor del módulo de corte, $n_{\mathrm{g}}$ : exponente del módulo de corte, $K_{\mathrm{b}}$ : factor del módulo volumétrico, $n_{\mathrm{b}}$ : exponente del módulo volumétrico, $\phi$ : ángulo de fricción interna y $c$ : cohesión 
Los módulos de corte máximo $G_{\max }$ y volumétrico máximo $B_{\max }$, según sea el caso, se calculan a partir del correspondiente factor $K$, exponente $n$, presión media efectiva $p^{\prime}=\left(\sigma^{\prime}{ }_{1}+2 \sigma_{3}\right) / 3$ donde $\sigma^{\prime}{ }_{1}$ y $\sigma^{\prime}{ }_{3}$ son los esfuerzos efectivos mayor y menor respectivamente y la presión atmosférica $P_{\mathrm{a}}=100 \mathrm{kPa}$.

Para el enrocado, el ángulo de fricción del material se calcula en función de la tensión media efectiva $p$ ' y presión atmosférica $P_{\text {a }}$ viene dado por:

$\phi=-3.034 \ln \left(p^{\prime} / P_{\mathrm{a}}\right)+47.3$

donde $\phi_{\max }=48^{\circ}$. El módulo de corte máximo se expresa como:

$G_{\max }=\rho V_{\mathrm{s}}^{2}$

donde de mediciones de onda de corte en enrocado compactado se tiene que:

$V_{\mathrm{s}}=673\left(\sigma_{\mathrm{v}}^{\prime} / 500\right)^{0.2}>530 \mathrm{~m} / \mathrm{s}$

donde $\sigma^{\prime}$, es el esfuerzo efectivo vertical en $\mathrm{kPa}$.

Para modelar el comportamiento dinámico de los materiales (a excepción de la roca, la cual se considera elástica durante el análisis dinámico) se calibró el modelo de amortiguamiento histérico sigmoidal sig3 en base a curvas de degradación disponibles en la literatura técnica consistentes para estos tipos de materiales (Liao et al. (2013) para la transición y Rollins et al. (1998) para los enrocados). La calibración en FLAC3D se realizó mediante simulaciones numéricas de ensayos de corte simple cíclicos calculando la degradación del módulo de corte máximo $\left(G / G_{\max }\right)$ respecto de la deformación angular para un determinado rango de presiones de confinamiento.

\section{Análisis estático}

Para obtener el estado tensional de la presa al término de su construcción, se simuló en FLAC3D la construcción de la presa en etapas de $3 \mathrm{~m}$ hasta su altura máxima. Cada etapa se resuelve hasta alcanzar el equilibrio, y a continuación se actualizan las propiedades de los materiales en función de la presión media efectiva. Durante la simulación, los materiales fueron modelados de acuerdo con el modelo constitutivo Mohr-Coulomb de FLAC3D. La resistencia del material de enrocado, en términos del ángulo de fricción, se asignó en función de la presión de confinamiento, similar a lo propuesto por Leps (1970) para enrocados.

La Figura 2 muestra las tensiones y desplazamientos al final de la construcción de la presa. Estos resultados se muestran en un corte transversal, aproximadamente ubicado al centro de la presa.
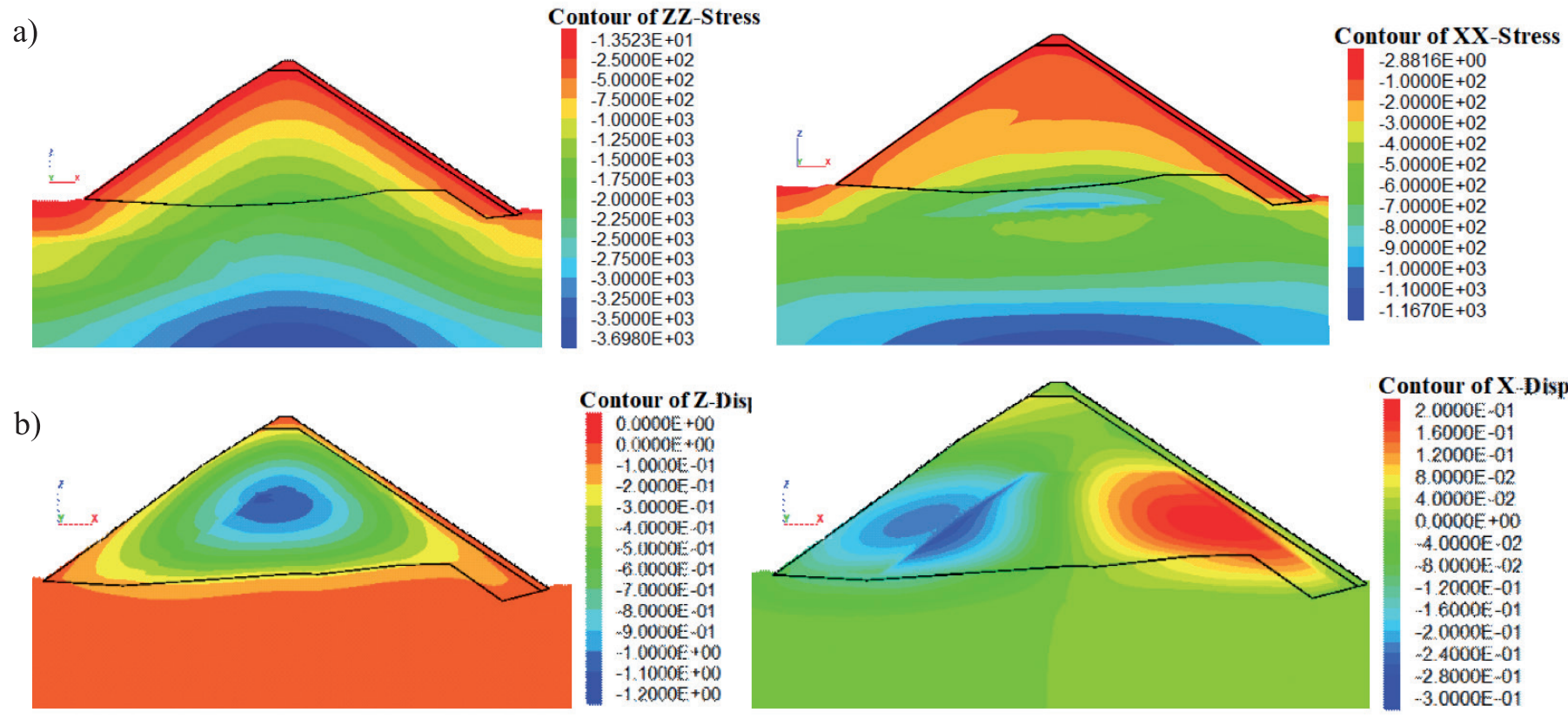

Contour of $X$-Displ

Figura 2: Contornos, al final de la construcción de la presa, de: a) distribución de tensiones verticales y horizontales y b) desplazamientos verticales y horizontales 


\section{Análisis dinámico}

El análisis dinámico se realizó bajo dos escenarios: lleno y vacío. Como se mencionó anteriormente, para una condición de embalse lleno se concluyó que no era necesario modelar la pantalla, puesto que el agua en contacto con el talud de aguas arriba confiere tal confinamiento al material inmediatamente detrás, que éste aumenta su rigidez y su resistencia, generando menores deformaciones. Por otra parte, para la condición de embalse vacío se concluyó que la pantalla de hormigón debe ser modelada durante la simulación dinámica, ya que sin ésta el talud de aguas arriba se desplazaría libremente, violando las condiciones de borde que impone el plinto. Lo anterior, dado que la pantalla se apoya sobre el talud y está restringida de desplazamiento libre en la dirección del plinto.

Cabe destacar que dentro de la modelación se consideró una interfaz friccional entre la pantalla de hormigón y el material de relleno $2 \mathrm{~B}$, de modo que la pantalla de hormigón no restringe totalmente los desplazamientos de los rellenos.

Las condiciones de borde dinámicas durante la simulación corresponden a campo libre (free field) en los lados del modelo y a bordes absorbentes en la base de éste.

\section{Peligro sísmico}

Para incorporar la señal sísmica al modelo, se efectúo el escalamiento espectral del sismo de Valparaíso de 1985 al espectro de diseño del sitio del embalse para el nivel sísmico MCE. Este sismo fue seleccionado dado su poder destructivo y duración relativamente corta. El escalamiento espectral del registro se efectuó mediante el programa RSPMatch09 (Al Atik y Abrahamson, 2010).

En la Figura 3 se muestra el ajuste espectral de las componentes horizontales y los registros de aceleraciones resultantes.

\section{Implementación de la pantalla de hormigón}

Para implementar la pantalla de hormigón y la condición impuesta por el plinto, se utilizaron elementos estructurales que incorpora FLAC3D (Cundall, 2016). La pantalla fue modelada por medio de una serie de elementos estructurales tipo Shell. Los Shell son elementos planos con 6 grados de libertad por cada uno de sus 3 nodos, admitiendo esfuerzos

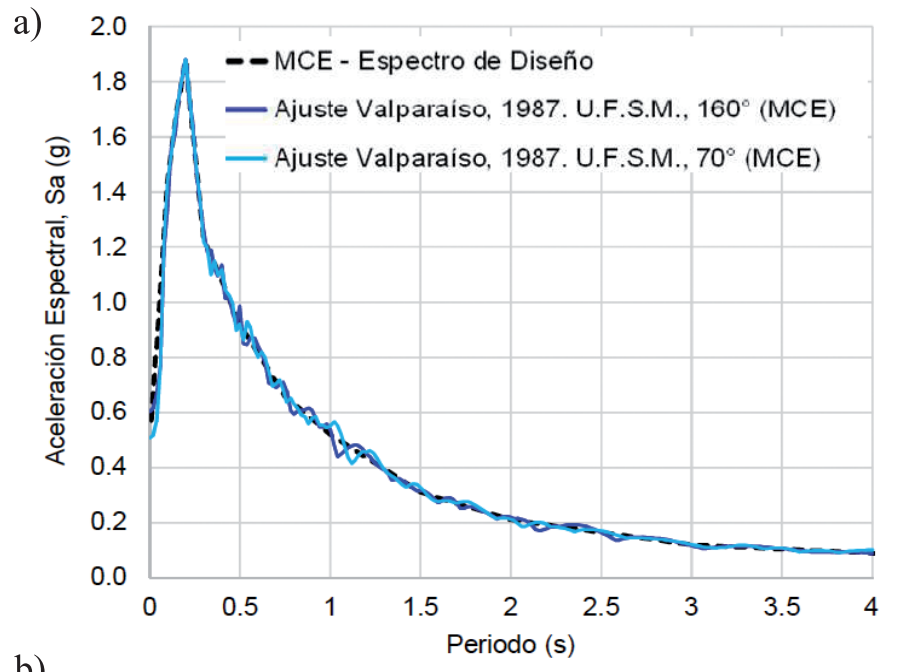

b)

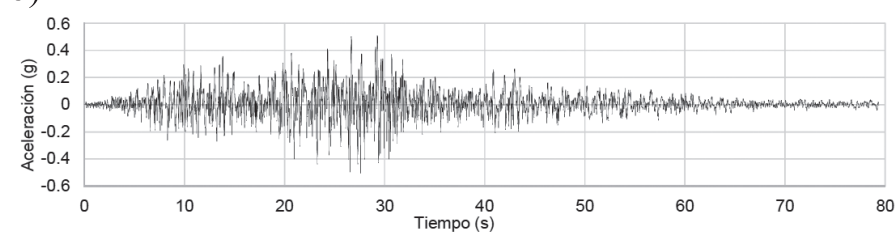

c)

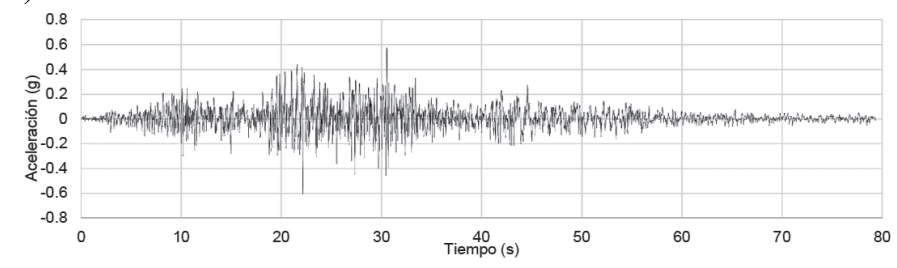

Figura 3: a) Ajuste espectral registro sísmico Valparaíso de 1985, b) componente $70^{\circ}$ y c) componente $160^{\circ}$

en el plano y fuera de este. Cada Shell se modeló como un material isotrópico linealmente elástico, mediante los parámetros: módulo de elasticidad, igual a $2.35 \mathrm{GPa}$, y la razón de Poisson igual a 0.2. Así el comportamiento tensión-deformación de la pantalla queda controlado por estas propiedades. Por otro lado, el espesor de la pantalla varía en función de la altura de la presa, desde $0.53 \mathrm{~m}$ en la base hasta $0.30 \mathrm{~m}$ en su altura máxima. La densidad adoptada del hormigón es de $2.4 \mathrm{t} / \mathrm{m}^{3}$.

La interacción entre los elementos estructurales y la grilla de la presa se modeló puramente friccional en las direcciones locales $x$ e $y$ de la pantalla, y elástica con límite de tensión en la dirección perpendicular. Además, y para simular las juntas entre losas, se incorporaron rótulas en las losas cada 15 m (ver Figura 4). Éstas permiten la rotación relativa entre ellas, pero solo en torno al eje $x$.

Para simular el efecto que impone el plinto durante la simulación dinámica, en la fundación de la pantalla se 
implementaron elementos estructurales del tipo cable, los que constan de 2 nodos y tres grados de libertad por nodo. Estos elementos se comportan como un material elastoplástico que puede deformarse sólo axialmente. De esta manera, cuando un nodo de la pantalla alcanzó un desplazamiento por sobre $10 \mathrm{~cm}$ en la dirección del plinto, se incorporó un elemento cable con alta resistencia a la compresibilidad y muy baja resistencia a la tracción. Esta configuración permite simular la interacción entre la pantalla y el plinto, de modo de restringir su desplazamiento más allá de los límites del plinto, pero sin impedirle que pueda volver a deformarse en la otra dirección. De lo contrario, la pantalla se podría deslizar libremente entrando en contacto con la roca, situación que no reflejaría la realidad del problema.

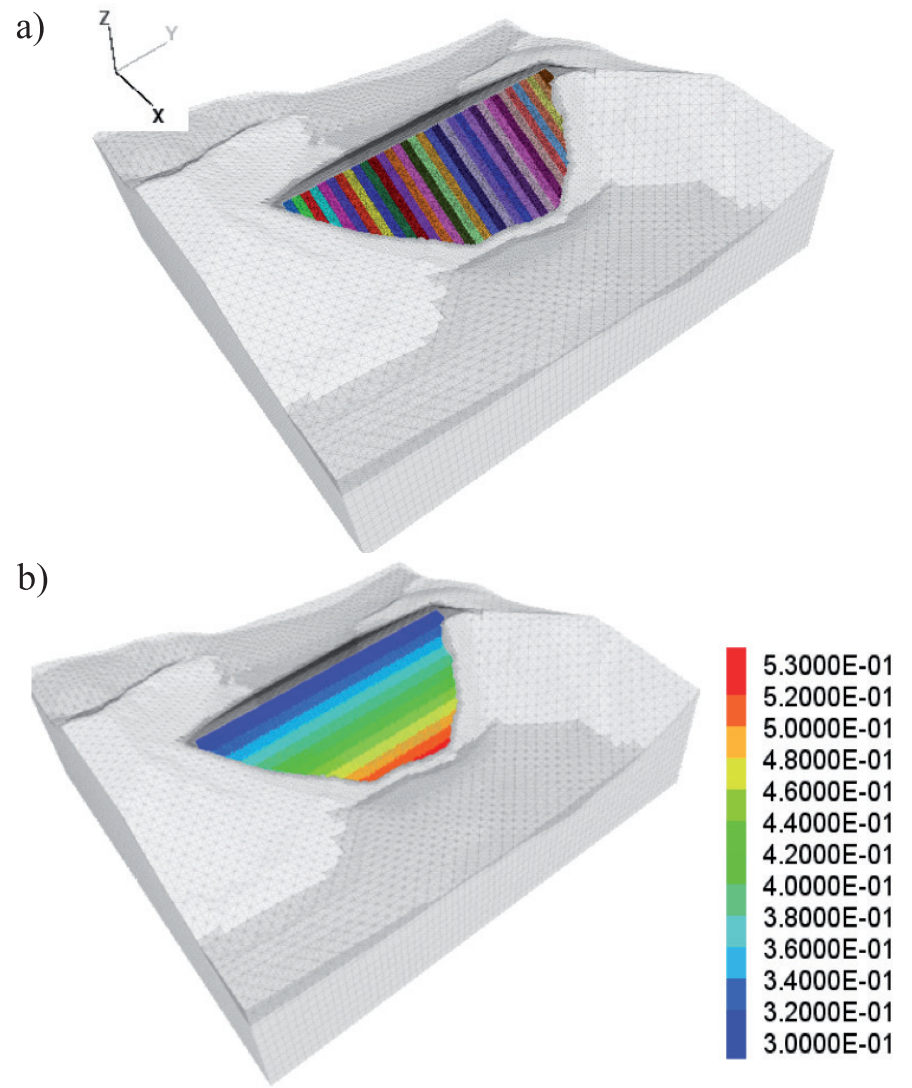

Figura 4: Pantalla de hormigón. a) Losas de elementos Shell con $15 \mathrm{~m}$ de ancho $\mathrm{y} \mathrm{b}$ ) espesor en metros de la pantalla a lo largo del muro

\section{Resultados}

Para validar la correcta transmisión de las ondas a través del modelo numérico, se verificó el ajuste de los espectros de aceleraciones de las señales de entrada y las rescatadas en la roca de la superficie del modelo. Esto permite concluir que las ondas sísmicas se propagan de forma correcta a través de la malla y que las condiciones de borde seleccionadas son las adecuadas para la trasmisión de estas. A continuación, se presentan los resultados para ambos modelos (con y sin pantalla), en términos de desplazamientos para el muro de enrocados y esfuerzos de momentos para la pantalla.

En las Figuras 5 y 6 se comparan los desplazamientos transversales al coronamiento (eje $\mathrm{x}$ ) y verticales de cada configuración al final del sismo, respectivamente.

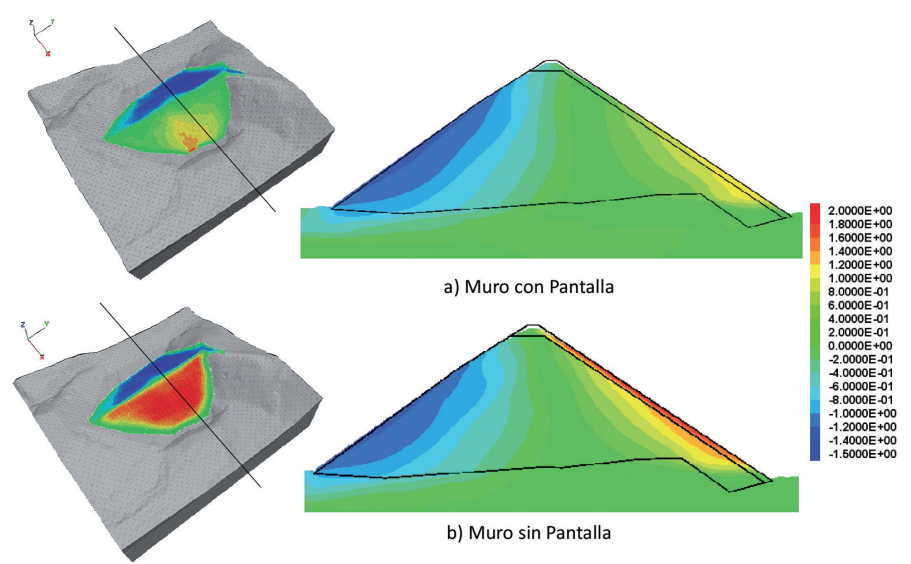

Figura 5: Resultados al final del registro sísmico. Contornos de distribución de desplazamientos en $\mathrm{x}$ en la presa

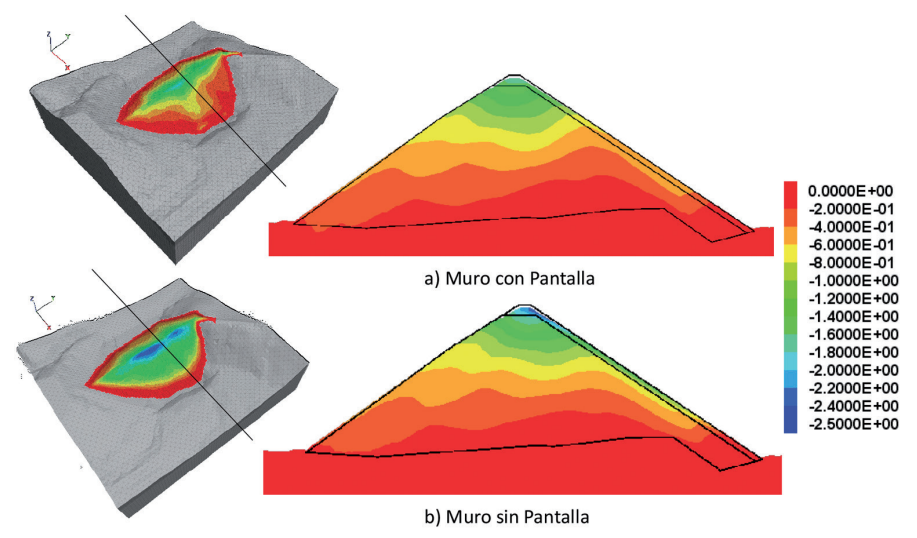

Figura 6: Resultados al final del registro sísmico. Contornos de distribución de asentamientos en la presa

Respecto a las deformaciones transversales a la presa, se observa que para ambos casos la cara del talud aguas abajo exhibe una distribución uniforme de los desplazamientos en función de la profundidad, con un valor máximo de $1.5 \mathrm{~m}$ en la superficie del talud. En cambio, en el talud aguas arriba, tanto la magnitud como la distribución de los desplazamientos presentan diferencias. Para el caso en 
que se modela la pantalla, las deformaciones alcanzadas son aproximadamente $1 \mathrm{~m}$ menor en relación con la otra configuración, lo anterior dado que el material en la cara de talud (2B-3A) desarrolla un mayor nivel de deformación. Esto sugiere que la pantalla proporciona la rigidez suficiente para restringir el nivel de deformación de este material. Notar que, para el caso con pantalla, los máximos desplazamientos horizontales en planta se concentran hacia el estribo izquierdo, lo que indica que en este sector la pantalla sufrió un mayor nivel de deformación producto de la acción dinámica.

En relación a los asentamientos, se observa que en la cara del talud en contacto con la pantalla se generan menores valores de deformación. Los resultados en planta muestran que, para el caso con pantalla, los asentamientos verticales máximos se generan en aproximadamente la misma zona donde se produjeron las máximas deformaciones horizontales.

Es importante destacar que las deformaciones, tanto horizontales como verticales, en el cuerpo de la presa no cambian significativamente con la presencia o ausencia de la pantalla de hormigón, puesto que los efectos sobre las deformaciones son sólo locales, tal como se mencionó anteriormente y se observa en las Figuras 5 y 6.

Los resultados presentados en la Figura 7 muestran que, para el caso modelado con pantalla, los máximos momentos se concentran en los bordes en contacto con el plinto como efecto de la interacción dinámica entre ambos. Observándose que los máximos valores corresponden a aproximadamente $450 \mathrm{kNm}$ y $2250 \mathrm{kNm}$, para los momentos flectores $M_{\mathrm{x}} \mathrm{y} M_{\mathrm{y}}$ respectivamente. Notar que, para el caso modelado con pantalla, el momento $M_{\mathrm{x}}$ no es un valor continuo, como lo es el momento $M_{\mathrm{y}}$. Esto se debe a la implementación de las juntas, las cuales no transmiten momento, haciendo que en los contornos se muestren valores nulos a lo largo de la junta.

El post análisis realizado en el caso sin pantalla implicó rescatar los desplazamientos de la cara aguas arriba del muro e imponerlos en los nodos de la pantalla. Los resultados muestran que los momentos flectores, en ambas direcciones del modelo sin pantalla, alcanzan valores menores a $10 \mathrm{kNm}$. Estos resultados podrían verse intensificados a consecuencia de la deformación relativamente subparalela y homogénea del material de

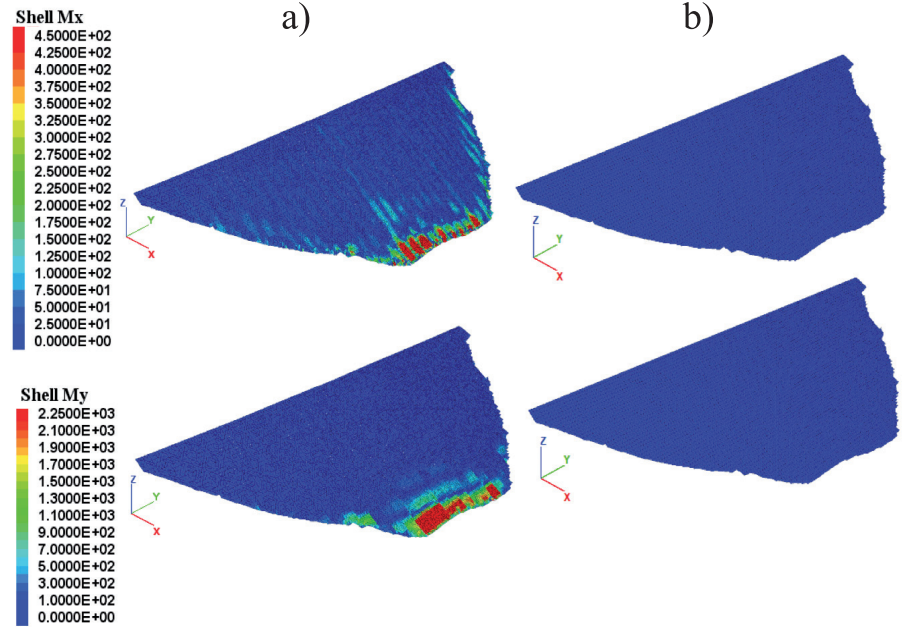

Figura 7: Resultados al final del registro sísmico. Contornos de distribución de momentos absolutos en la dirección $x$ e $y$. a) Pantalla incluida durante la modelación y b) pantalla mediante post análisis

transición sobre el talud aguas arriba, en el caso modelado sin pantalla. Es evidente que los resultados antes expuestos muestran los efectos de no modelar adecuadamente la pantalla de hormigón. Especial impacto se observa en que se podría subestimar significativamente los momentos producidos en la pantalla de hormigón.

\section{Conclusiones}

En este estudio se han expuesto los efectos de no incorporar la pantalla de hormigón en el análisis dinámico de presas CFRD. La pantalla de hormigón durante la simulación dinámica restringe el nivel de deformación sobre el talud de aguas arriba, lo que se esperaría bajo condiciones reales, puesto que la rigidez y resistencia de la pantalla impide que se desarrollen mayores deformaciones. En relación a lo anterior, si bien el alcance de este estudio no contempló un análisis de sensibilidad sobre las propiedades de la pantalla, es factible presumir que estas sí tienen influencia sobre el nivel de deformación en el muro.

Los resultados dinámicos muestran que la metodología de post análisis, utilizada para el caso sin pantalla, y utilizada ampliamente en la práctica, subestima significativamente los momentos flectores resultantes respecto de cuando esta es modelada. Este efecto podría verse intensificado, dado el patrón de deformación que presenta el material de transición, cuando la pantalla está ausente.

Finalmente, los resultados indican que las técnicas de modelación aquí presentadas, tales como; la interacción 
friccional pantalla-muro, la discretización e interacción entre las losas, la simulación de la acción del plinto, permiten de forma adecuada simular las condiciones que impondría la estructura de hormigón y el plinto a este tipo de embalses. Además, es importante considerar que la modelización 3D del problema juega un rol primordial en la respuesta dinámica de la pantalla, puesto que la aplicación bidireccional del sismo permite capturar en ambos ejes la interacción entre las losas como su interacción con la acción del plinto en todo su borde, que un estudio bidimensional no podría capturar.

\section{Agradecimientos}

Los autores agradecen a la Dirección de Obras Hidráulica del Ministerio de Obras Públicas de Chile por permitir usar los resultados de este proyecto en este estudio.

\section{Referencias}

Al Atik, L. and Abrahamson, N. (2010). An improved method for nonstationary spectral matching. Earthquake Spectra 26(3), 601-617
Cundall, P.A. (2016). FLAC3D: Fast Lagrangian Analysis of Continua in 3 Dimensions, Version 5.0, Itasca Consulting Group, Inc., Minneapolis, MN, USA

Leps, T. (1970). Review of shearing strength of rockfill. Journal of the Soil Mechanics and Foundations Division 96(4), 11591170

Liao, T., Massoudi, N., McHood, M., Stokoe, K.H., Jung, M.J. and Menq, F.Y. (2013). Normalized shear modulus of compacted gravel. 18 $8^{\text {th }}$ International Conference on Soil Mechanics and Geotechnical Engineering, Paris, France, vol. 2, 1535-1538

Rollins, K.M., Evans, M.D., Diehl, N.B. and Daily, W.D. (1998). Shear modulus and damping relationships for gravels. Journal of Geotechnical and Geoenvironmental Engineering 124(5), 396405 